\title{
Trapped Antihydrogen in Its Ground State
}

\author{
G. Gabrielse, ${ }^{1, *}$ R. Kalra, ${ }^{1}$ W. S. Kolthammer, ${ }^{1}$ R. McConnell, ${ }^{1}$ P. Richerme, ${ }^{1}$ D. Grzonka, ${ }^{2}$ W. Oelert, ${ }^{2}$ T. Sefzick, ${ }^{2}$ \\ M. Zielinski, ${ }^{2}$ D. W. Fitzakerley, ${ }^{3}$ M. C. George, ${ }^{3}$ E. A. Hessels, ${ }^{3}$ C. H. Storry, ${ }^{3}$ M. Weel, ${ }^{3}$ A. Müllers, ${ }^{4}$ and J. Walz ${ }^{4}$
}

(ATRAP Collaboration)

\author{
${ }^{1}$ Department of Physics, Harvard University, Cambridge, Massachusetts 02138, USA \\ ${ }^{2}$ IKP, Forschungszentrum Jülich GmbH, 52425 Jülich, Germany \\ ${ }^{3}$ Department of Physics and Astronomy, York University, Toronto, Ontario M3J 1P3, Canada \\ ${ }^{4}$ Institut für Physik, Johannes Gutenberg Universität and Helmholtz Institut Mainz, D-55099 Mainz, Germany
}

(Received 27 December 2011; published 16 March 2012)

\begin{abstract}
Antihydrogen atoms $(\overline{\mathrm{H}})$ are confined in an Ioffe trap for 15-1000 s-long enough to ensure that they reach their ground state. Though reproducibility challenges remain in making large numbers of cold antiprotons $(\bar{p})$ and positrons $\left(e^{+}\right)$interact, $5 \pm 1$ simultaneously confined ground-state atoms are produced and observed on average, substantially more than previously reported. Increases in the number of simultaneously trapped $\overline{\mathrm{H}}$ are critical if laser cooling of trapped $\overline{\mathrm{H}}$ is to be demonstrated and spectroscopic studies at interesting levels of precision are to be carried out.
\end{abstract}

DOI: 10.1103/PhysRevLett.108.113002

The original proposal to use cold $\bar{p}$ and $e^{+}$to produce $\overline{\mathrm{H}}$ atoms that could be confined in a magnetic trap for precise spectroscopy [1] and gravitational measurements [2] is being actively pursued. Such spectroscopy (demonstrated later with $\mathrm{H}$ [3]) could compare $\overline{\mathrm{H}}$ and $\mathrm{H}$ at a higher precision than the most stringent $C P T$ tests with leptons [4] and baryons [5]. Cold $\overrightarrow{\mathrm{H}}$ production [6,7], at rates increased by driving forces [8], recently led to a demonstration of almost one $\overline{\mathrm{H}}$ atom per trial trapped for many seconds [9]. $\overline{\mathrm{H}}$ spectroscopy may eventually use only one trapped atom, but attaining interesting levels of precision will initially require many more simultaneously trapped atoms [10].

When ATRAP produced the first $\overline{\mathrm{H}}$ atoms in an Ioffe trap designed to confine them [11], no trapped atoms were observed. In an average of $N$ trials, ATRAP's detector could detect an average of $12 / \sqrt{N}$ simultaneously trapped $\overline{\mathrm{H}}$ atoms per trial at a $3 \sigma$ level. Increases in the detection efficiency, the number of trials, and/or the number of simultaneously trapped $\overline{\mathrm{H}}$ were thus required. The latter is the most attractive since more simultaneously trapped $\overline{\mathrm{H}}$ are needed to demonstrate laser cooling, and then precise laser and microwave spectroscopy.

To increase the likelihood that cold $\overline{\mathrm{H}}$ would be produced, we cooled the electrodes of the traps containing $\bar{p}$ and $e^{+}$to $1.2 \mathrm{~K}$ [12], demonstrated that $e^{+}$or $e^{-}$stored within these thermalized at $20 \mathrm{~K}$ [13], and prepared up to

Published by the American Physical Society under the terms of the Creative Commons Attribution 3.0 License. Further distribution of this work must maintain attribution to the author(s) and the published article's title, journal citation, and DOI.
PACS numbers: 37.10.De, 36.10.-k, 41.20.-q, 41.90.+e

$10^{7}$ cold $\bar{p}$ for producing cold $\overline{\mathrm{H}}$ [13]. This Letter reports using $10^{6} \bar{p}$ (over an order of magnitude more than used for any previous attempt to trap $\overline{\mathrm{H}}$ ) to produce $5 \pm 1$ simultaneously trapped $\overline{\mathrm{H}}$ atoms on average. The $\overline{\mathrm{H}}$ energies are below $375 \mathrm{mK}$ (with the low energy expressed in temperature units), and confinement times between 15 and $1000 \mathrm{~s}$ ensure that they are in their ground state. The number of confined $\overline{\mathrm{H}}$ produced compares favorably to a very recent report [9] of $0.7 \pm 0.3$ atoms, with energies below $500 \mathrm{mK}$ stored from 50 to $2000 \mathrm{~s}$, produced using many fewer $\bar{p}$. Our demonstration that more $\bar{p}$ produce more trapped $\overline{\mathrm{H}}$ suggests that it may be possible to further scale up the number of simultaneously trapped $\overline{\mathrm{H}}$ using the $10^{7} \bar{p}$ and many more $e^{+}$currently available.

Each of the 20 trials in this demonstration take up to $2 \mathrm{~h}$. One hour is used to accumulate and cool $\bar{p}$ and $30 \mathrm{~min}$ to accumulate and cool $e^{+}$. Up to $30 \mathrm{~min}$ is used to bring the $\bar{p}$ and $e^{+}$into position, ramp up the Ioffe trap, form $\overline{\mathrm{H}}$ atoms, and look for trapped $\overline{\mathrm{H}}$.

Similar methods accumulate $\bar{p}$ and $e^{+}$for all $\overline{\mathrm{H}}$ experiments [14]. Within a $B=3.7$ T magnetic field, we accumulate $10^{6} \bar{p}$ from 6 to 8 pulses of $3 \times 10^{7} \bar{p}$ delivered with a $5 \mathrm{MeV}$ energy approximately every $100 \mathrm{~s}$ by CERN's antiproton decelerator (AD). The $\bar{p}$ slow in a thin Be degrader window, are trapped within cylindrical Penning trap electrodes (Fig. 1), and thermalize via collisions with trapped $e^{-}$that are then ejected. The $e^{+}$from a ${ }^{22} \mathrm{Na}$ source are trapped after they collide with gas molecules [15], and are transported though a $9.5 \mathrm{~m}$ magnetic guide to enter the trap (from the right in Fig. 1). The trap electrodes are biased to form a nested Penning trap [16] [e.g., Fig. 2(a)]. Approximately $3 \times 10^{7}$ thermalized $e^{+}$ are positioned in the trap center, with $10^{6} \bar{p}$ at the bottom of 


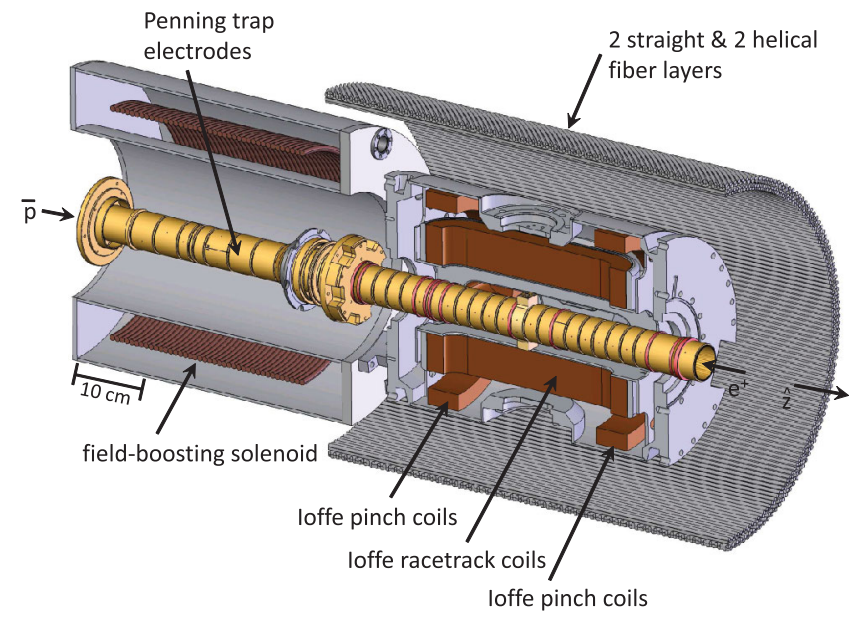

FIG. 1 (color). Electrodes and coils produce Penning traps (to store $\bar{p}$ and $e^{+}$) and an Ioffe trap [22] (to store $\overline{\mathrm{H}}$ ). Much of the vacuum enclosure and cooling system is hidden to make the traps and detectors visible. An external solenoid (not shown) adds a $1 \mathrm{~T}$ magnetic field along the trap axis $\hat{\mathbf{z}}$ which is vertical.

the well to the right. After $B$ is reduced to $1 \mathrm{~T}$, currents approaching $100 \mathrm{~A}$ are introduced into the pinch and racetrack coils [11]. This creates a $375 \mathrm{mK}$ Ioffe trap for an $\overline{\mathrm{H}}$ atom in its ground state [with equipotentials in Fig. 2(b)] to confine low-field-seeking $\overline{\mathrm{H}}$ formed with sufficiently low kinetic energy.

$\overline{\mathrm{H}}$ atoms form via a three-body interaction of a $\bar{p}$ and two $e^{+}[16,17]$. In a search for the most efficient production of cold $\overline{\mathrm{H}}$, the 20 trials differ primarily in the driving force applied to make the $\bar{p}$ gain enough energy to pass through the $e^{+}$[8]. To maintain resonance with the anharmonic $\bar{p}$ center-of-mass oscillation as the $\bar{p}$ oscillation energy
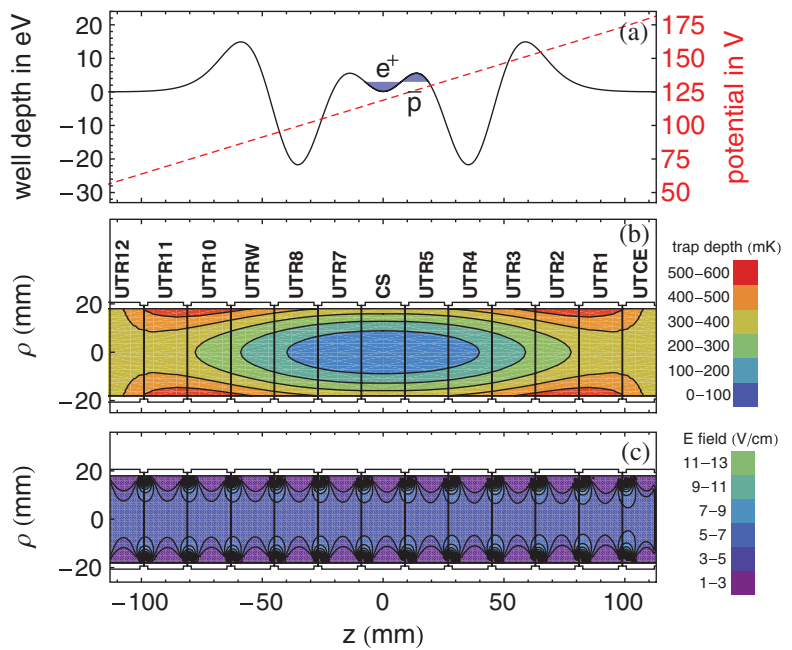

FIG. 2 (color). (a) Potentials along the center axis used to contain (solid line) and remove (dashed line) charged particles. (b) Electrode cross sections with equipotential energy contours for a low-field-seeking, ground state $\overline{\mathrm{H}}$ in the Ioffe trap. (c) Axial electric field contours used to clear $\bar{p}$ and $e^{+}$before trapped $\overline{\mathrm{H}}$ are detected. The trap axis is vertical. increases, some trials apply a driving force with a frequency spectrum broadened by noise for up to $10 \mathrm{~min}$. Other trials use a coherent drive, chirped in frequency for a duration of $2 \mathrm{~ms}$ to $15 \mathrm{~min}$. (See Fig. 3 of [8] and [18].)

$\overline{\mathrm{H}}$ production and trapping continues for the $2 \mathrm{~ms}$ to 15 min that the $\bar{p}$ and $e^{+}$interact in the various trials. An $\overline{\mathrm{H}}$ atom stays confined as long as its radiative decay takes it to another low-field-seeking state whose kinetic energy is less than the Ioffe trap well depth for the state. The $\bar{p}$ and $e^{+}$are then cleared out by axial electric fields of about $\pm 5 \mathrm{~V} / \mathrm{cm}$ [Figs. 2(a) and 2(c)]. These fields, parallel to the trap axis, are chosen to be much stronger than any stray fields that could otherwise trap a $\bar{p}$ given the $B$ field. For a $\bar{p}$ to be trapped directly by the Ioffe trap, its cyclotron magnetic moment would require a $140 \mathrm{eV}$ cyclotron energy-much larger than the axial energy that could be transferred to cyclotron motion by $\bar{p}-\bar{p}$ collisions.

The $\overline{\mathrm{H}}$ remaining in the trap are released by quenching the superconducting racetrack coils of the Ioffe trap. The quench is caused by a heat pulse from a resistor (early trials) or by exceeding the critical current (later trials).

The minimum $\overline{\mathrm{H}}$ storage time ranges from 15 to $60 \mathrm{~s}$ (the time between the application of the clearing electric field and the quench). However, in many trials the $\overline{\mathrm{H}}$ storage time could be as long as the $1000 \mathrm{~s}$ between when $\overline{\mathrm{H}}$ production starts and the quench. The integral [Fig. 3(b)] of the electromotive force (emf) induced [Fig. 3(a)] in the field-boosting solenoid in Fig. 1 identifies the $1 \mathrm{~s}$ time interval during which the radial Ioffe trap well depth is reduced so that $93 \%$ of a uniform distribution of $\overline{\mathrm{H}}$ energies can escape the trap. In this interval, the signal from escaping $\overline{\mathrm{H}}$ annihilating on an electrode competes with the cosmic ray background.

The $\bar{p}$ annihilation pions (and cosmic rays) are detected using 4 layers of $3.8 \mathrm{~mm}$ scintillating fibers (2 straight and 2 helical in Fig. 1). Made of BICRON BCF-12, with a peak emission wavelength of $435 \mathrm{~nm}$ and an attenuation length of $2.7 \mathrm{~m}$, these 784 fibers are located outside the trap vacuum enclosure and the Dewar that cools it (not shown in Fig. 1). A double layer of 24 large scintillating paddles $1 \mathrm{~m}$ high surrounds the Dewar for the 1 Tesla
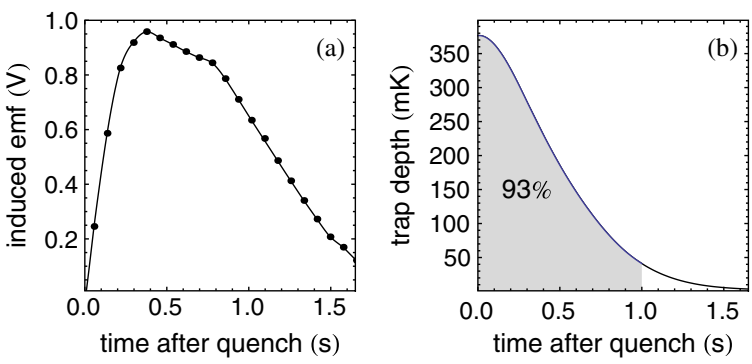

FIG. 3 (color online). (a) The emf induced across the fieldboosting solenoid as the Ioffe racetrack coil quenches. (b) Deduced Ioffe trap well depth for ground state $\overline{\mathrm{H}}$ shows that if these fill the trap uniformly in energy $93 \%$ will escape within $1 \mathrm{~s}$ after the quench. 
superconducting solenoid (on a $66 \mathrm{~cm}$ radius, outside the view of Fig. 1). Coincidences between the fibers and paddles detect $\bar{p}$ annihilations with an efficiency of 54\% and a cosmic ray background of $41 \mathrm{~Hz}$.

A time-stamped record of the fibers and paddles triggered near the time of the quench is acquired at a rate up to $10^{3}$ events per second-much higher than the observed count rate. The probabilities that 4096 scintillator combinations are triggered by $\bar{p}$ annihilations and by cosmic ray are measured using the annihilations of $3 \times 10^{5} \bar{p}$ spilled radially and $3.5 \times 10^{5}$ cosmic ray events. A Monte Carlo simulation shows that selecting the 256 scintillator combinations for which the ratio of these probabilities are greater than 4.55 optimizes the signal-to-noise ratio-providing enough signal while minimizing the effect of background fluctuations. This choice reduces the background to $1.7 \mathrm{~Hz}$ from $41 \mathrm{~Hz}$ while decreasing the $\bar{p}$ detection efficiency from $54 \%$ to $33 \%$.

As described, the method used to make large $\bar{p}$ and $e^{+}$ plasmas interact is varied from trial to trial. No clear favorite has yet emerged, and the interaction of the plasmas varies noticeably even for trials intended to be identical. Accordingly, we sum the detector counts for all of the 20 trials carried out during the $2011 \mathrm{AD}$ run. The background averages down to allow a small signal from trapped $\overline{\mathrm{H}}$ to become visible, suggesting that some or all of the methods produce trapped $\overline{\mathrm{H}}$.

Figure 4(a) shows the sum of the detector counts for the 20 trials in $1 \mathrm{~s}$ intervals, including the interval in which quenches emptied the Ioffe trap [dark gray (blue)]. The pronounced peak, when divided by the detector efficiency, shows that $105 \pm 21 \overline{\mathrm{H}}$ atoms were trapped in the $375 \mathrm{mK}$ quadrupole Ioffe trap. This corresponds to an average of $5 \pm 1$ simultaneously trapped $\overline{\mathrm{H}}$ per trial, stored in the trap for between 15 and $1000 \mathrm{~s}$. This signal is 6 standard deviations above what is expected from the observed background [right vertical scale in Fig. 4(a)], indicating that there is only 1 chance in $10^{7}$ that the signal in the central channel is a fluctuation of the cosmic background. The counts in the $1 \mathrm{~s}$ intervals before and after the central signal interval are consistent with the measured statistical background.

Figure 4(c) shows the sum of 20 control trials made by quenching the Ioffe trap when no $\overline{\mathrm{H}}$ are trapped. It shows that the sudden flux change from quenching the trap does not induce false coincidence signals that could be misinterpreted as being from $\overline{\mathrm{H}}$ atoms.

The best of the $20 \overline{\mathrm{H}}$ trials illustrates current challenges and future possibilities. The count average and fluctuations outside of the central $1 \mathrm{~s}$ time interval are consistent with the other trials. However, the counts in the central bin (corresponding to $39 \pm 8 \overline{\mathrm{H}}$ atoms when the detection efficiency is included) are much higher than the average. Sometimes we produce more $\overline{\mathrm{H}}$ atoms and sometimes fewer, owing to our inability to precisely control the
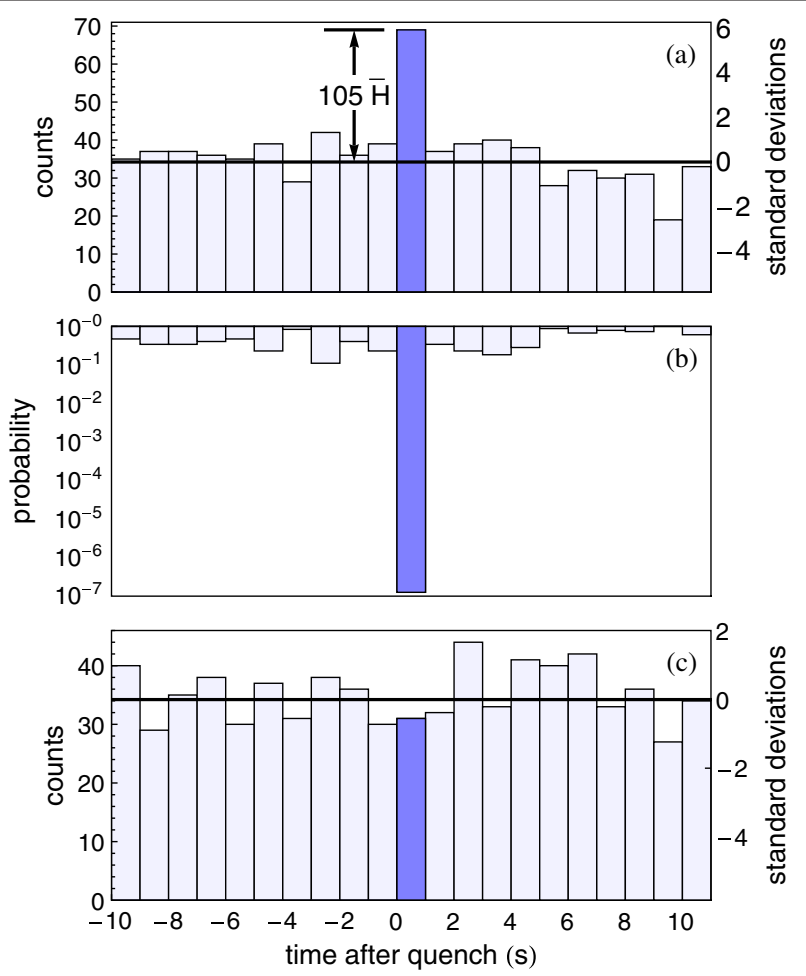

FIG. 4 (color online). (a) Detector counts in $1 \mathrm{~s}$ intervals for 20 trials. The radial Ioffe trap field turns off and releases trapped $\overline{\mathrm{H}}$ between $t=0$ and $1 \mathrm{~s}$. The counts in this interval above the average cosmic ray counts (solid line) correspond to 105 trapped $\bar{p}$ for our detection efficiency. (b) Probability that cosmic rays produce the observed counts or more. (c) Quenching the Ioffe trap generates no false signals in 20 control trials.

interaction of the $\bar{p}$ and $e^{+}$, even in "identical" trials. Identical trials also produce slightly different $\bar{p}$ and $e^{+}$ plasma diameters and differences in the rate at which $\bar{p}$ and $e^{+}$escape the nested trap. If we analyze our trials without the best one (though we have no justification for discarding it), the average number of simultaneously trapped $\overline{\mathrm{H}}$ per trial is $3.5 \pm 0.7$. This is consistent with the average for all 20 trials, with a statistical significance of $4 \sigma$ (a probability of less than $3 \times 10^{-4}$ of this being a background fluctuation). Better control of the interaction of the large $\bar{p}$ and $e^{+}$ plasmas in a substantial magnetic gradient should produce the large number of trapped $\overline{\mathrm{H}}$ in every trial.

To realize the long-time goal of precise $\overline{\mathrm{H}}$ spectroscopy [1], the $\overline{\mathrm{H}}$ atoms must be in their ground state. However, essentially all the $\overline{\mathrm{H}}$ produced as $\bar{p}$ and $e^{+}$interact in a nested Penning trap are in highly excited Rydberg states (demonstrated earlier using ATRAP's field ionization method [8]). These highly excited, guiding-center atoms [17] are high-field-seeking states that cannot be trapped.

The trapped atoms must thus come from the small $\overline{\mathrm{H}}$ fraction that the earlier field ionization measurements showed were produced with radii smaller than $0.14 \mu \mathrm{m}$ [14]. (This corresponds to a principal quantum number $n \approx 50$, though $n$ is not a very good quantum number for 
large $B$.) Such atoms were shown to have chaotic $e^{+}$orbits [14] owing to comparable strengths of the nonlinear Coulomb and magnetic forces on the $e^{+}$. Some are apparently weak-field-seeking states, trapped via diamagnetic forces that are large for large $B$.

To remain in the trap for 15 to $1000 \mathrm{~s}$, the radiating $\overline{\mathrm{H}}$ must remain in low-field-seeking states. Simulations $[19,20]$ suggest this can happen, presumably because the angular momentum quantum number $m$ does not change on average, and $\Delta m= \pm 1$ in a single radiative decay.

An overestimate of the time required for an $n=50$ state to decay to $n=1$ is the slowest radiation path, that from one circular state $(m=l=n-1)$ to another. The radiation rate for these states goes as $1 / n^{5}$ and the $n=50$ circular state has a $30 \mathrm{~ms}$ lifetime. Rate equations describe a cascade to the ground state that takes about $0.5 \mathrm{~s}$. The actual cascade time is shorter given that fields and collisions mix in states with lower $l$ quantum numbers that radiate much more rapidly than circular states. Thus $\overline{\mathrm{H}}$ detected after a $15-1000 \mathrm{~s}$ storage time are in their ground state.

Trapped $\overline{\mathrm{H}}$ make it possible to compare their gravitational force $\kappa M g$ to the familiar $M g$ on a $\mathrm{H}$ atom [2]. Atoms created at the magnetic minimum on axis acquire $\kappa M g h$ of energy in free fall to the magnetic maximum of the trap, with $h=10.6 \mathrm{~cm}$. The atoms will escape a magnetic trap with an energy depth $W$ (375 $\mathrm{mK}$ in temperature units here) unless $|\kappa| \leq W /(M g h)=3000$. For our trials, a $2 \sigma$ level signal is present during the time that the radial well depth is reduced from 375 to $350 \mathrm{mK}$, establishing that $|\kappa|<200$. Improved limits will be possible with more trapped $\overline{\mathrm{H}}$, laser cooling and probing of the $\overline{\mathrm{H}}$ spatial distribution [2]. It may eventually be possible to exceed the limit $|\kappa-1|<$ $1 \times 10^{-6}$ set by the consistency to better than 1 part in $10^{10}$ of $\bar{p}$ and $p$ cyclotron clocks [5]. Such clocks would have different gravitational redshifts if the gravitation force differs by a factor of $\kappa$ for $\bar{p}$ and $p$ [21].

In conclusion, more simultaneously trapped $\overline{\mathrm{H}}$ atoms can be formed when large $\bar{p}$ and $e^{+}$plasmas are used, despite the ongoing difficulties of controlling the interaction of large plasmas in a stable and reproducible way. The approximately $105 \overline{\mathrm{H}}$ atoms observed to be trapped in a $375 \mathrm{mK}$, quadrupole Ioffe trap correspond to an average of 5 simultaneously trapped $\overline{\mathrm{H}}$ atoms per trial. We are optimistic that increases in the number of trapped $\overline{\mathrm{H}}$ atoms are coming. Progress in manipulating and controlling the large and cold $\bar{p}$ plasmas seems likely to continue. Lower plasma temperatures seem feasible. A new Ioffe trap just being assembled should make it possible to make the $\bar{p}$ and $e^{+}$interact more efficiently. Even more simultaneously trapped $\overline{\mathrm{H}}$ should be possible if these methods can be adapted for use with the 10 times more $\bar{p}$ that we have shown can be accumulated, and the ELENA upgrade to CERN's AD should make larger numbers of $\bar{p}$ available.

We are grateful to CERN for providing $5-\mathrm{MeV} \bar{p}$ and some support for W. O. The NSF and AFOSR of the U.S., the BMBF, DFG, and DAAD of Germany, and the NSERC, CRC, and CFI of Canada support this work.

*ATRAP Spokesperson. gabrielse@physics.harvard.edu

[1] G. Gabrielse, in Fundamental Symmetries, edited by P. Bloch, P. Pavlopoulos, and R. Klapisch (Plenum, New York, 1987) pp. 59-75.

[2] G. Gabrielse, Hyperfine Interact. 44, 349 (1989).

[3] C. L. Cesar, D. G. Fried, T. C. Killian, A. D. Polcyn, J. C. Sandberg, I. A. Yu, T. J. Greytak, D. Kleppner, and J. M. Doyle, Phys. Rev. Lett. 77, 255 (1996).

[4] R. S. Van Dyck, Jr., P. B. Schwinberg, and H. G. Dehmelt, Phys. Rev. Lett. 59, 26 (1987).

[5] G. Gabrielse, A. Khabbaz, D. S. Hall, C. Heimann, H. Kalinowsky, and W. Jhe, Phys. Rev. Lett. 82, 3198 (1999).

[6] M. Amoretti et al., Nature (London) 419, 456 (2002).

[7] G. Gabrielse, N. S. Bowden, P. Oxley, A. Speck, C. H. Storry, J. N. Tan, M. Wessels, D. Grzonka, W. Oelert, G. Schepers, T. Sefzick, J. Walz, H. Pittner, T. W. Hänsch, and E. A. Hessels, Phys. Rev. Lett. 89, 213401 (2002).

[8] G. Gabrielse, N. S. Bowden, P. Oxley, A. Speck, C. H. Storry, J. N. Tan, M. Wessels, D. Grzonka, W. Oelert, G. Schepers, T. Sefzick, J. Walz, H. Pittner, T. W. Hänsch, and E. A. Hessels, Phys. Rev. Lett. 89, 233401 (2002).

[9] G. B. Andresen et al., Nature Phys. 7, 558 (2011).

[10] C. Zimmerman and T. Hänsch, Hyperfine Interact. 76, 47 (1993).

[11] G. Gabrielse, P. Larochelle, D. Le Sage, B. Levitt, W. S. Kolthammer, R. McConnell, P. Richerme, J. Wrubel, A. Speck, M. C. George, D. Grzonka, W. Oelert, T. Sefzick, Z. Zhang, A. Carew, D. Comeau, E. A. Hessels, C. H. Storry, M. Weel, and J. Walz, Phys. Rev. Lett. 100, 113001 (2008).

[12] J. Wrubel, G. Gabrielse, W. S. Kolthammer, P. Larochelle, R. McConnell, P. Richerme, D. Grzonka, W. Oelert, T. Sefzick, M. Zielinski, J. S. Borbely, M. C. George, E. A. Hessels, C. H. Storry, M. Weel, A. Mullers, J. Walz, and A. Speck, Nucl. Instrum. Methods Phys. Res., Sect. A 640, 232 (2011).

[13] G. Gabrielse, W.S. Kolthammer, R. McConnell, P. Richerme, R. Kalra, E. Novitski, D. Grzonka, W. Oelert, T. Sefzick, M. Zielinski, D. Fitzakerley, M. C. George, E. A. Hessels, C. H. Storry, M. Weel, A. Mullers, and J. Walz, Phys. Rev. Lett. 106, 073002 (2011).

[14] G. Gabrielse, Adv. At. Mol. Opt. Phys. 50, 155 (2005).

[15] C. M. Surko, M. Leventhal, and A. Passner, Phys. Rev. Lett. 62, 901 (1989).

[16] G. Gabrielse, S. L. Rolston, L. Haarsma, and W. Kells, Phys. Lett. A 129, 38 (1988).

[17] M. Glinsky and T. O’Neil, Phys. Fluids B 3, 1279 (1991).

[18] G. B. Andresen et al., Phys. Rev. Lett. 106, 025002 (2011).

[19] T. Pohl, H. R. Sadeghpour, Y. Nagata, and Y. Yamazaki, Phys. Rev. Lett. 97, 213001 (2006).

[20] F. Robicheaux, Phys. Rev. A 73, 033401 (2006).

[21] R. J. Hughes and M. H. Holzscheiter, Phys. Rev. Lett. 66, 854 (1991).

[22] D. E. Pritchard, Phys. Rev. Lett. 51, 1336 (1983). 